\title{
46-km-Long Raman Amplified Hybrid Double-Bus Network With Point and Distributed Brillouin Sensors
}

\author{
Montserrat Fernandez-Vallejo, David Olier, Ander Zornoza, Rosa Ana Perez-Herrera, Silvia Diaz, Cesar Elosua, \\ Candido Bariain, Alayn Loayssa, and Manuel Lopez-Amo, Senior Member, IEEE
}

\begin{abstract}
We experimentally demonstrate a 46-km hybrid network that combine point and distributed Brillouin sensors. The proposed sensor network multiplexes low-cost intensity point sensors based on fiber-optic tapers, which are able to measure vibrations in the 0.01 to $50 \mathrm{~Hz}$ frequency range. The sensor network with a double-bus is a low noise configuration, which offers a higher optical signal to noise ratio and dynamic range than a single-bus. Thus, the number of sensors to be multiplexed could increase or we could reach further distances. The system also deploys remote distributed Raman amplification to extend the sensing range.
\end{abstract}

Index Terms-Brillouin distributed sensing, fiber Bragg grating (FBG), fiber optics sensors, Raman amplification, wavelength division multiplexing (WDM).

\section{INTRODUCTION}

$\mathbf{F}$ IBER optic sensors (FOS) exhibit meaningful benefit when are compared with traditional sensors such as small size, high sensibility, flexibility, embeddability, EMI immunity, electrical isolation, the possibility of withstanding harsh environments, and being multiplexed in sensor networks. For this reason, FOS is a promising technology in structural health monitoring systems (SHM). [1]

Structural health monitoring is a tool to measure and analyze an infrastructure with the purpose of giving maintenance and predicting ongoing dangers or future accidents. This technology is currently utilized in the monitoring of civil infrastructures such as building, piles, bridges, highway traffic monitoring, pipelines, tunnels, or dams.

To have an accurate control of these huge systems under test two principal points have to be taken into account:

First, it can be necessary to measure different parameters such as strain, stress, displacement, pressure, load, acceleration, temperature, gas concentrations, or vibrations [1]. In particular, the detection of low frequency vibrations (from 10 to $0.1 \mathrm{~Hz}$ ) needs to be monitored in pipelines, civil structures, buildings, etc. [1], [5]. Fiber-optic-taper based sensor employed as transducers is

Manuscript received February 04, 2011; accepted March 10, 2011. This work was supported in part by the Spanish Ministerio de Educación y Ciencia under Projects TEC2007-67987-C02-02 and TEC2010-20224-C02-01. The associate editor coordinating the review of this manuscript and approving it for publication was Prof. Brian Culshaw.

The authors are with the Department of Electrical and Electronic Engineering, Public University of Navarra, Pamplona, Navarra 31006, Spain (e-mail: montserrat.fernandez@unavarra.es; dolier@unavarra.es; ander.zornoza@unavarra.es; rosa.perez@unavarra.es; silvia.diaz@unavarra.es; cesar.elosua@unavarra.escba@unavarra.es; alayn.loayssa@unavarra.es; mla@unavarra.es).

Color versions of one or more of the figures in this paper are available online at http://ieeexplore.ieee.org.

Digital Object Identifier 10.1109/JSEN.2011.2131128

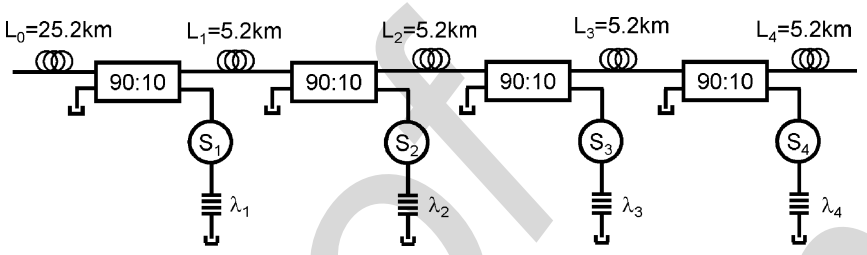

Fig. 1. Previous single bus topology.

a suitable technology for doing these tasks. These kinds of sensors are able to measure a number of environmental parameters [6] or gases [7].

Second, it is necessary to choose between "point," "quasi-distributed," and "distributed" sensors. The fiber optic point sensors are enabled to measure at specific points in the structure. A quasi-distributed sensor system is composed of different point sensors multiplexed in a fiber. Finally, distributed fiber optic sensors are particularly suitable for large structural monitoring applications since all the segments of an optical fiber act as sensors, and therefore the perturbations within various segments of the structure can be sensed [2], [3].

The three major distributed sensor techniques are: optical time domain reflectometry, sensors based on Raman, and Brillouin Scattering. The most appropriate technique for distributed sensing is based on Brillouin scattering because it provide simultaneous information of temperature and strain along the length of a standard low-cost telecommunication optical fiber [3].

Brillouin scattering can be stimulated by the use of a probe wave; thus, higher signal-to-noise ratios are attainable. Moreover, long distance measurements are possible because standard low-loss single-mode fibers are used and optical amplification can be deployed to further enhance of the reach of these systems [4].

Furthermore, long-range sensing networks for point sensors are now possible using Er-doped fiber or Raman optical amplification [8], [9].

We have previously reported a long-range hybrid network with point and distributed Brillouin sensors using Raman amplification which offered a great advantage: the fiber length deployed to interconnect and multiplex point sensors also serves as distributed sensor [10]. Nevertheless, the sensor network with a single bus topology, shown in Fig. 1, presents high noise levels.

In this paper, we propose an experimentally demonstrate an improved sensor network for multiplexing the point sensors based on a double-bus topology. The low noise configuration offers a great improvement in the signal-to-noise ratio; thus, 


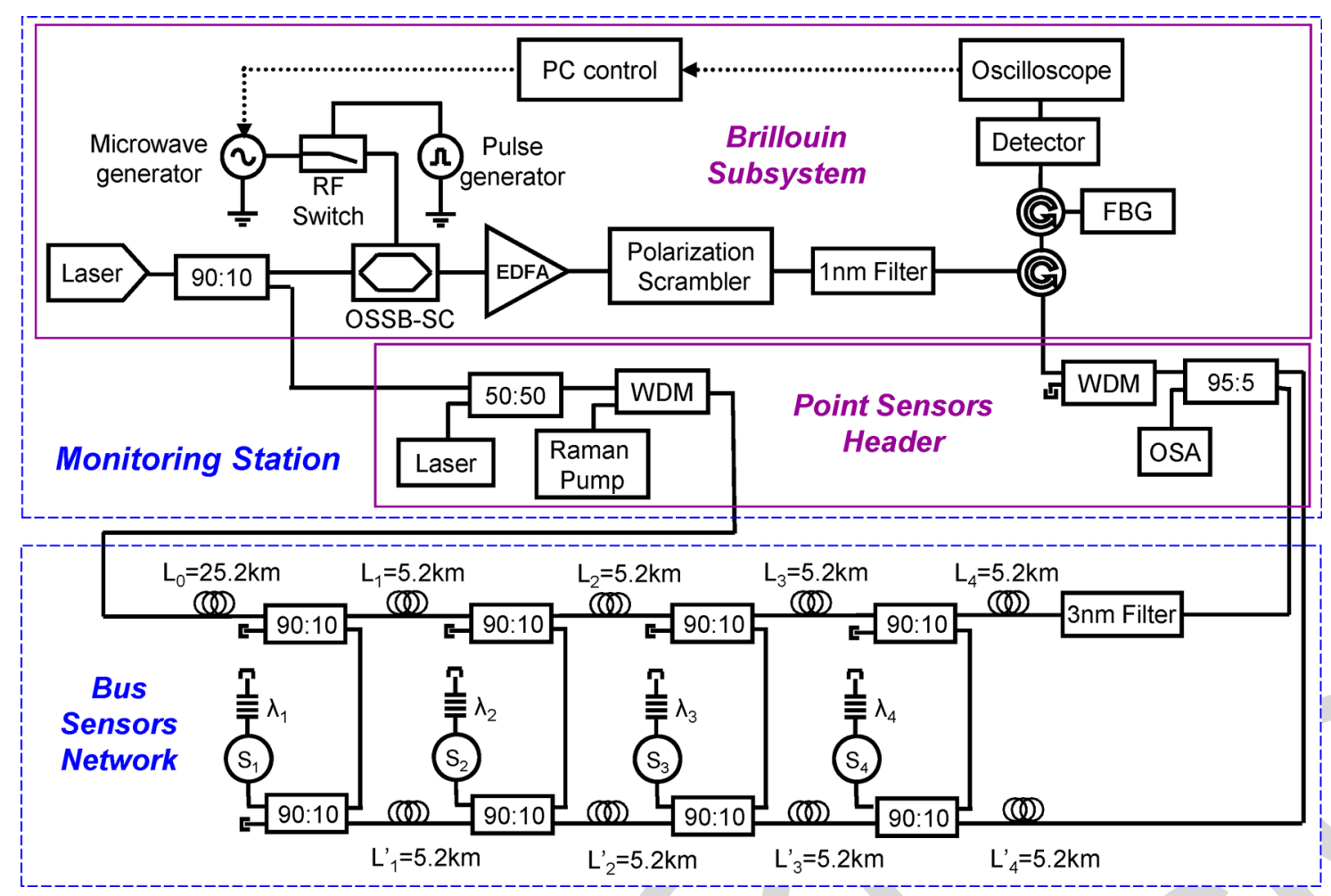

Fig. 2. Hybrid sensor network with point and distributed optical sensors.

the number of sensors to be multiplexed could increase or we could reach further distances.

\section{MethodOLOGY}

The experimental setup for the hybrid sensor network is shown in Fig. 2. It can be divided into two different parts: the sensor network itself and the monitoring station equipment.

The sensor network has a double-bus topology with a total length of $46 \mathrm{~km}$. Four-point sensors are wavelength-division multiplexed (WDM). Each sensor is addressed by a narrow-bandwidth FBG at a unique wavelength. Although the launched signals reaches all the sensors, the FBG ensures that each sensor returns only its information to the point sensor header after passing through the sensor a second time.

In the monitoring station are generated the signals needed for interrogation of the point sensors and for the Brillouin distributed measurements. The Brillouin measurement system is based on the Brillouin optical time-domain analysis (BOTDA) principle in which a pulsed pump and a continuous (CW) Sokes waves counterpropagate in the fiber under test (FUT).

A detailed explanation of the point sensors network subsystem and the Brillouin distributed sensing subsystem is presented in Sections II-A and II-B, respectively.

\section{A. Point Sensors Network Subsystem}

It is proposed a WDM network for point sensors multiplexing over $46 \mathrm{~km}$ of single-mode fiber used also for distributed sensing. The sensor network is composed by $46 \mathrm{~km}$ of standard single-mode fiber (SMF) and it has a double-bus topology based on [11] and [12]. It is used for both distributed sensing and point sensors multiplexing simultaneously.
The point sensors network subsystem includes eight directional couplers (90:10 $\pm 0.3 \%$ ratios) to perform power distribution among the sensors. The distance between two adjacent couplers is about $5.2 \mathrm{~km} \mathrm{SMF}$. Both buses are identical with four couplers each one in order to wavelength-division multiplexing (WDM) the point vibration sensors.

The sensors, addressed by four fiber Bragg gratings centered at $\lambda_{1}=1539.8 \mathrm{~nm}, \lambda_{2}=1538.47 \mathrm{~nm}, \lambda_{3}=1533.9 \mathrm{~nm}$, and $\lambda_{4}=1535.36 \mathrm{~nm}$, are separated by $5.2-\mathrm{km}$ fiber spans. The grating wavelengths are located on the short wavelength side of the peak of Raman gain profile.

A Raman pump laser generates distributed amplification in the sensor bus using the first span of 25.2-km SMF, thus Raman gain is maximized in the bus and the fiber sensor network range is extended. The Raman pump power is launched into the fiber bus by using a fiber wavelength division multiplexer (WDM). It is able to launch up to $3.2 \mathrm{~W}$ into the single-mode fiber at $1445 \mathrm{~nm}$.

The signal used to interrogate the point sensors is a tunable laser (1460-1580 nm) with a spectral linewidth of $5 \mathrm{MHz}$.

The Raman pump and the signal are located at one end of the fiber outputs of the monitoring station. Another WDM is used at the other end of the bus to prevent the residual Raman pump from re-entering the monitoring station. Also the optical spectral analyzer (OSA) is located in the other head end. All the free terminations on the double-bus are refractive-index-matched to eliminate undesirable reflections.

The point vibration sensors multiplexed are based on fiberoptic taper sensors employed as transducers to measure mechanical vibrations. They show some advantages, as their simple fabrication process, low-cost and versatility in the number of mea- 
surable parameter [13]. The tapers were implemented by elongating the fiber during a fusion process, [14]. The transmitted optical power of the taper depends on its bending radius: as it decreases, the transmitted power decreases as well [15], so it can be used to vibrations just following the power variations. In this case, the optical signal crosses the taper back and forth, because the sensors are used in reflective configurations. Thus, there must be a compromise between the sensors' sensitivity, the induced losses and the maximum measuring length because although the sensors have double sensitivity the also have double losses.

\section{B. Brillouin Distributed Sensing Subsystem}

In the Brillouin distributed sensor, the output of a tunable laser source is first divided in two branches, so that the same light source is used for pump and stokes generation. In the upper branch a Dual Parallel Mach-Zehnder (DPMZ) biased at minimum transmission generates a pulsed optical single side band suppressed carrier modulation (POSSB-SC). The pulsing is achieved in the electrical domain using the RF shaping proposed in [16] so as to avoid leakage. The POSSB-SC signal is then applied to the sensing fiber as pump pulse after an active polarization scrambler is used to compensate the polarization sensitivity of SBS. Then, a 1-nm wide optical filter tuned to the wavelength of the POSSB-SC signal is inserted to reduce the amplified spontaneous emission (ASE) noise from the erbium-doped amplifier.

In the lower branch the laser output is directly used to provide the probe wave. No modulation at all is needed in this branch. In order to avoid any crosstalk from the point sensors interrogation system a fiber Bragg grating filter is used before detection. Note that the double-bus system makes no difference in the Brillouin sensing subsystem compared to the setup in [10].

\section{EXPERIMENTAL RESULTS}

It is experimentally demonstrated the hybrid double-bus network with point and distributed Brillouin sensors using Raman amplification following the design explained in previous section.

\section{A. Point Sensors Measurements}

In this subsection, we show the measurements obtained utilizing the point sensor inside the multiplexing network.

The fiber-optics sensors were fabricated as was explained in [10]. When vibration is applied to these taper sensors, the fiber is bent in the stretched area, giving a curvature's change, and then, a variation in the transmitted power. To characterize the sensor's response, we applied vibrations of different frequency, from $0.01 \mathrm{~Hz}$ to $100 \mathrm{~Hz}$; amplitude, from $0.025 \mathrm{~mm}$ to $3.25 \mathrm{~mm}$, and waveform, sinusoidal, square, triangular, and sawtooth waves. The results can be compared to the ones offered by commercial vibrometers with $\mathrm{mm} / \mathrm{s}$ RMS units [10].

Fig. 3(a) presents the spectrum for the four point sensors when Raman pump power is $1 \mathrm{~W}$. Amplification of the Brillouin signal and the signal used to interrogate the point sensors have been taking into account to select the optimum pump power. The output reflected signals reached $-24 \mathrm{dBm}$ and an OSNR of $55 \mathrm{~dB}$.
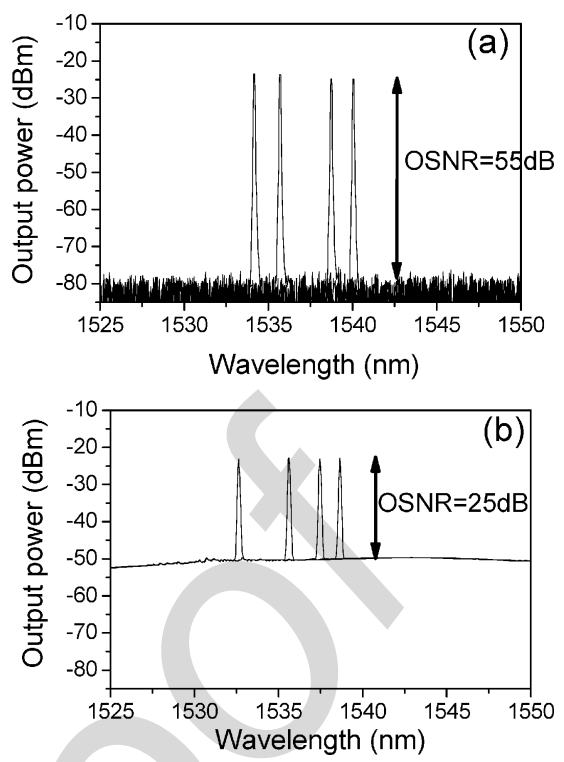

Fig. 3. Optical spectrum for the four point sensors. (a) Double-bus topology. (b) Single-bus topology.

The advantages of optical amplifier inclusion in sensor multiplexing networks are well-known. However, optical amplifier also involves constraints as a new noise source: amplified spontaneous scattering (ASS) if Raman amplification is used. For this reason, to optimizing sensor multiplexing networks behavior will be necessary to deplete the ASS. The double-bus topology presented obeys this important requirement.

Although the double-bus topology is more complicated than the used in a previous work where the sensor network was composed by a single bus [10], it offers a great appeal: it decrease the ASS noise level $30 \mathrm{~dB}$ below the values achieved by the single-bus topologies. The reduction of the ASS results in a great improvement of the OSNR. The single bus topology offers an OSNR of $27 \mathrm{~dB}$, while the double-bus topology OSNR reaches $55 \mathrm{~dB}$ as it is shown in Fig. 3. This enhancement is reached because in the single-bus topology, the backward amplified spontaneous-scattering (ASS) was co-propagating with the modulated signal and both were coupled to the OSA; however, with the double-bus topology the forward ASS propagates in the contrary direction than the signal, and thus only a bit amount of ASS reaches the OSA. Thus, with this low-noise configuration the number of sensors that could be multiplexed increases.

The main design parameter of the point sensors is the loss induced by the taper, which determines the sensitivity and the dynamic range. The losses induced by the sensor are $1.75 \mathrm{~dB}$, which were doubled in the experimental setup because the signal passes through each sensor twice, so the maximum losses are $3.5 \mathrm{~dB}$.

The double-bus topology has the advantage of obtaining an improvement in signal-to-noise ratio; thus, the dynamic range increases. The OSNR of this configuration reaches $55 \mathrm{~dB}$ : keeping in mind that the losses induced by the sensor are doubled because the signal passes twice through it, the insertion losses together with the dynamic range could be up to $27.5 \mathrm{~dB}$. For instance, in the case that the insertion losses were $3.5 \mathrm{~dB}$ to enhance the sensitivity, the dynamic range of the sensor could 


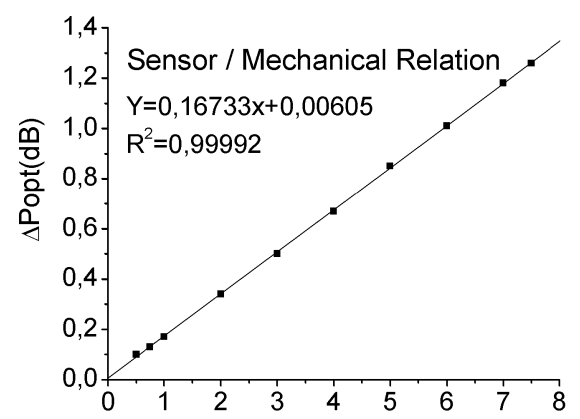

Peak to peak Vibration Amplitude, Dpp (mm)
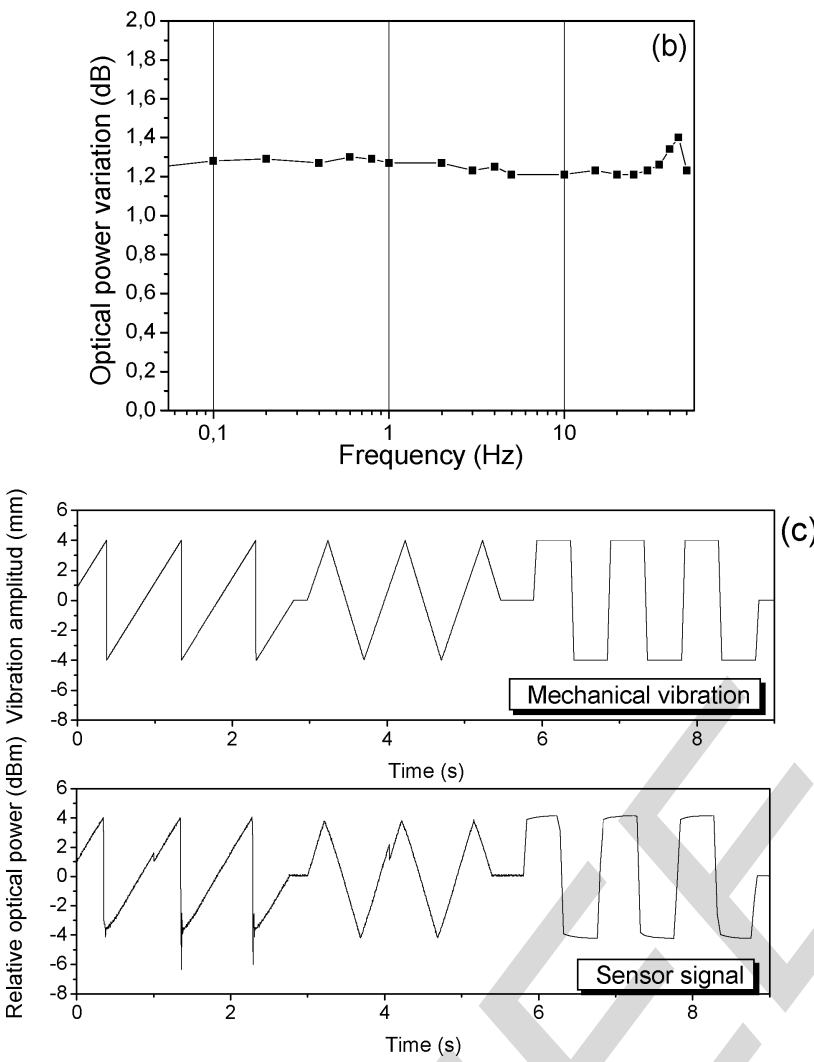

Fig. 4. Sensor's frequency, amplitude, and waveform responses for a sensor.

be up to $24 \mathrm{~dB}$ : in our case, they are $1.3 \mathrm{~dB}$ as a consequence of the design parameters of the sensor. For this reason, we could use sensor with higher dynamic range, add more point sensors in the network or reach further distances.

We have characterized the sensor behavior regarding two main parameters: frequency and amplitude response. To measure the frequency response, a sinusoidal wave with $6.5 \mathrm{~mm}$ peak-to-peak variation at different frequencies was applied. To analyze the amplitude response, a $45-\mathrm{Hz}$ vibration frequency was used and the amplitude was varied from 6.5 to $0.05 \mathrm{~mm}$ peak-to-peak. Fig. 4(a) shows that there is a linear relationship between the amplitude of the vibration and the variation in the sensor's signal. Regarding to the frequency response [Fig. 4(b)], it shows a resonance peak around $50 \mathrm{~Hz}$, so it might be used to operate with frequency below $30 \mathrm{~Hz}$. Fig. 4(c) depicts as different waveforms can be deployed as excitation signals: the response from the sensor follows the vibration signal from the vibrator.
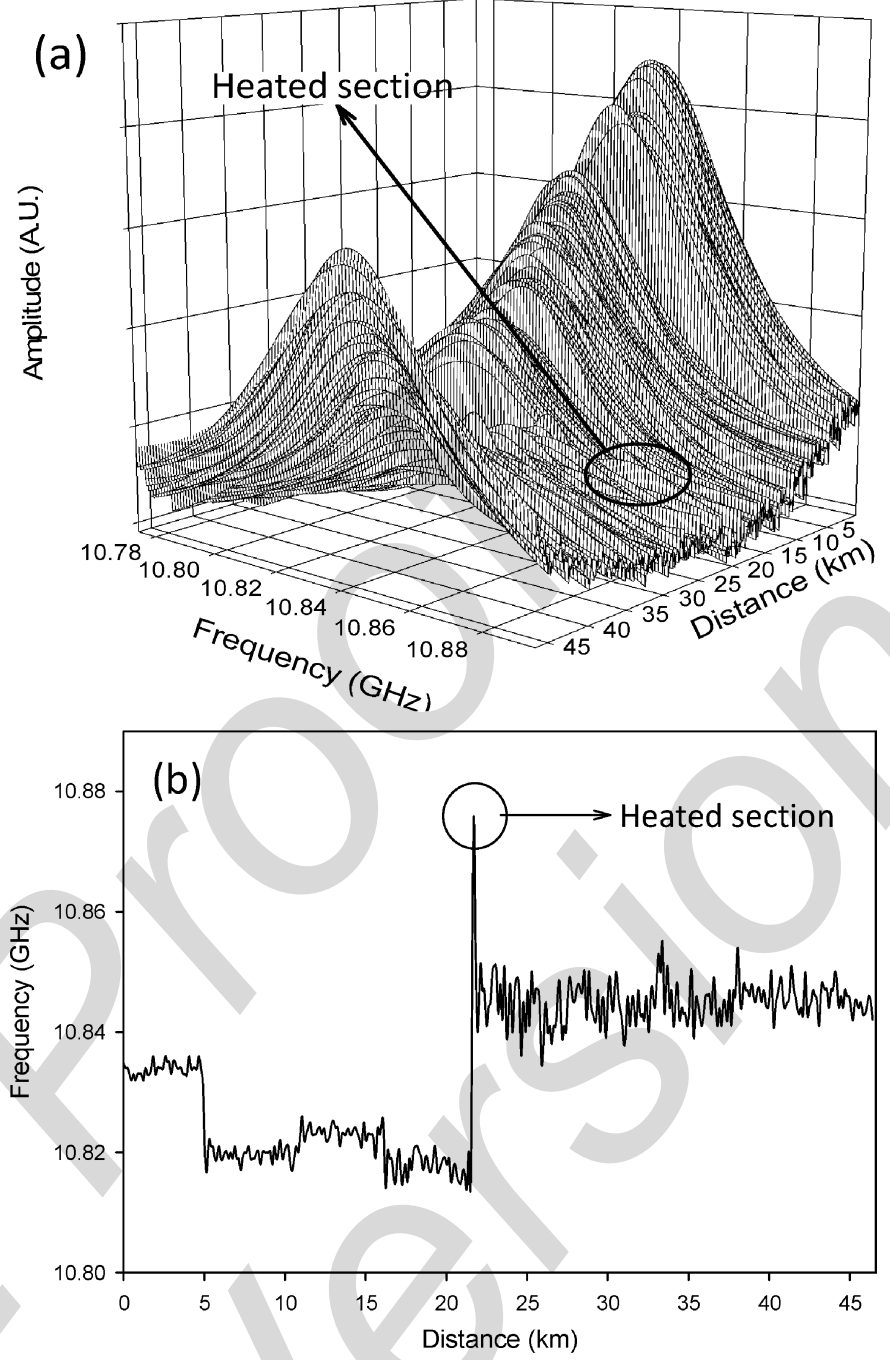

Fig. 5. (a) Distributed measurement of the Brillouin spectra. (b) Brillouin frequency shift of the whole fiber.

\section{B. Brillouin Distributed Temperature Measurements}

In the Brillouin distributed sensor we performed distributed temperature measurements having a $3-\mathrm{m}$ resolution. $200 \mathrm{~m}$ of the fiber at a distance of $25 \mathrm{~km}$ of the beginning were placed in a climatic chamber at $55{ }^{\circ} \mathrm{C}$ while the rest was held at room temperature. The RF was swept at 1-MHz steps and the traces were averaged 4000 times. The distributed measurement of the Brillouin spectra is shown in Fig. 5(a) and the Brillouin frequency shift of the whole fiber are shown in Fig. 5(b). Different types of fibers used in the bus sensors network and heated section are clearly distinguishable with a resolution of $4{ }^{\circ} \mathrm{C}$. This resolution was calculated in the worst case scenario: in the middle of the fiber, where the pump pulse power is minimum due to propagation loss and Raman amplification is not still noticeable.

\section{CONCLUSION}

A 46-km double-bus hybrid network that integrates point and distributed Brillouin sensors and uses Raman amplification has been implemented. The use of Raman amplification in BOTDA distributed sensors serves to compensate branching and fiber losses in the network and provides a spatial resolution of $3 \mathrm{~m}$. 
Furthermore, the simultaneous Raman amplification of the signals from the low-frequency (from 0.1 to $50 \mathrm{~Hz}$ ) vibration point sensors permits to place them as far as the end of the fiber bus at $46 \mathrm{~km}$ away from the interrogation equipment overcoming low power levels or backscattering noise problems.

\section{ACKNOWLEDGMENT}

The author would like to thank D. Leandro for his help.

\section{REFERENCES}

[1] H. -. Li, D. -. Li, and G. -. Song, "Recent applications of fiber optic sensors to health monitoring in civil engineering," Eng. Struct., vol. 26, pp. 1647-1657, 2004.

[2] E. Tapanes, "Fibre optic sensing solutions for real-time pipeline integrity monitoring," in Proc. Austral. Pipeline Ind. Assoc. Nat. Conv., 2001.

[3] C. Bariáin, I. R. Matías, F. J. Arregui, and M. López-Amo, "Experimental results towards development of humidity sensors by using a hygroscopic material on biconically tapered optical fibre," in Proc. SPIE - Int. Soc. Opt. Eng., Beijing, China, 1998, vol. 3555, pp. 95-105.

[4] C. Elosua, I. R. Matias, C. Bariain, and F. J. Arregui, "Volatile organic compound optical fiber sensors: A review," Sensors, vol. 6, pp. 1440-1465, 2006.

[5] M. Niklès, "Fibre optic distributed scattering sensing system: Perspectives and challenges for high performance applications," in Proc. SPIE - Int. Soc. Opt. Eng., Naples, Italy, 2007, vol. 6619, 66190D.

[6] J. M. Lopez-Higuera, Handbook of Optical Fibre Sensing Technology. New York: Wiley, 2002.

[7] M. N. Alahbabi, Y. T. Cho, and T. P. Newson, "150-km-range distributed temperature sensor based on coherent detection of spontaneous Brillouin backscatter and in-line Raman amplification," J. Opt. Soc. Amer. B, vol. 22, pp. 1321-1324, 2005.

[8] S. Diaz, S. Abad, and M. Lopez-Amo, "Fiber-optic sensor active networking with distributed erbium-doped fiber and Raman amplification," Laser Photon. Rev., vol. 2, pp. 480-497, 2008.

[9] S. Diaz, G. Lasheras, and M. Lopez-Amo, "WDM bi-directional transmission over $35 \mathrm{~km}$ amplified fiber-optic bus network using Raman amplification for optical sensors," Opt. Exp., vol. 13, pp. 9666-9671, 2005.

[10] A. Zornoza, R. A. Pérez-Herrera, C. Elosúa, S. Diaz, C. Bariain, A. Loayssa, and M. Lopez-Amo, "Long-range hybrid network with point and distributed Brillouin sensors using Raman amplification," Opt. Exp., vol. 18, pp. 9531-9541, 2010.

[11] S. Abad, M. López-Amo, J. M. López-Higuera, D. Benito, A. Unanua, and E. Achaerandio, "Single and double distributed optical amplifier fiber bus networks with wavelength-division multiplexing for photonic sensors," Opt. Lett., vol. 24, pp. 805-807, 1999.

[12] V. Montoya, M. López-Amo, and S. Abad, "Improved double-fiber-bus with distributed optical amplification for wavelength-division multiplexing of photonic sensors," IEEE Photon. Technol. Lett., vol. 12, no. 9, pp. 1270-1272, Sep. 2000.
[13] I. R. Matías, C. Fernández-Valdivielso, F. J. Arregui, C. Bariáin, and M. López-Amo, "Transmitted optical power through a tapered singlemode fiber under dynamic bending effects," Fiber Integr. Opt., vol. 22 , pp. 173-187, 2003.

[14] P. Datta, I. Matías, C. Aramburu, A. Bakas, M. López-Amo, and J. M. Otón, “Tapered optical-fiber temperature sensor," Microw. Opt Technol Lett., vol. 11, pp. 93-95, 1996.

[15] F. J. Arregui, I. R. Matias, C. Bariain, and M. Lopez-Amo, "Experimental design rules for implementing biconically tapered single mode optical fibre displacement sensors," in Proc. SPIE, 1998, vol. 3483, pp. $164-168$.

[16] A. Zornoza, D. Olier, M. Sagues, and A. Loayssa, "Brillouin distributed sensor using RF shaping of pump pulses," Meas. Sci. Technol., vol. 21, no. 9 , p. 094021,2010

Montserrat Fernandez-Vallejo, photograph and biography not available at the time of publication.

David Olier, photograph and biography not available at the time of publication.

Ander Zornoza, photograph and biography not available at the time of publication.

Rosa Ana Perez-Herrera, photograph and biography not available at the time of publication.

Silvia Diaz, photograph and biography not available at the time of publication.

Cesar Elosua, photograph and biography not available at the time of publication.

Candido Bariain, photograph and biography not available at the time of publication.

Alayn Loayssa, photograph and biography not available at the time of publication.

Manuel Lopez-Amo, photograph and biography not available at the time of publication. 


\title{
46-km-Long Raman Amplified Hybrid Double-Bus Network With Point and Distributed Brillouin Sensors
}

\author{
Montserrat Fernandez-Vallejo, David Olier, Ander Zornoza, Rosa Ana Perez-Herrera, Silvia Diaz, Cesar Elosua, \\ Candido Bariain, Alayn Loayssa, and Manuel Lopez-Amo, Senior Member, IEEE
}

\begin{abstract}
We experimentally demonstrate a 46-km hybrid network that combine point and distributed Brillouin sensors. The proposed sensor network multiplexes low-cost intensity point sensors based on fiber-optic tapers, which are able to measure vibrations in the 0.01 to $50 \mathrm{~Hz}$ frequency range. The sensor network with a double-bus is a low noise configuration, which offers a higher optical signal to noise ratio and dynamic range than a single-bus. Thus, the number of sensors to be multiplexed could increase or we could reach further distances. The system also deploys remote distributed Raman amplification to extend the sensing range.
\end{abstract}

Index Terms-Brillouin distributed sensing, fiber Bragg grating (FBG), fiber optics sensors, Raman amplification, wavelength division multiplexing (WDM).

\section{INTRODUCTION}

$\mathbf{F}$ IBER optic sensors (FOS) exhibit meaningful benefit when are compared with traditional sensors such as small size, high sensibility, flexibility, embeddability, EMI immunity, electrical isolation, the possibility of withstanding harsh environments, and being multiplexed in sensor networks. For this reason, FOS is a promising technology in structural health monitoring systems (SHM). [1]

Structural health monitoring is a tool to measure and analyze an infrastructure with the purpose of giving maintenance and predicting ongoing dangers or future accidents. This technology is currently utilized in the monitoring of civil infrastructures such as building, piles, bridges, highway traffic monitoring, pipelines, tunnels, or dams.

To have an accurate control of these huge systems under test two principal points have to be taken into account:

First, it can be necessary to measure different parameters such as strain, stress, displacement, pressure, load, acceleration, temperature, gas concentrations, or vibrations [1]. In particular, the detection of low frequency vibrations (from 10 to $0.1 \mathrm{~Hz}$ ) needs to be monitored in pipelines, civil structures, buildings, etc. [1], [5]. Fiber-optic-taper based sensor employed as transducers is

Manuscript received February 04, 2011; accepted March 10, 2011. This work was supported in part by the Spanish Ministerio de Educación y Ciencia under Projects TEC2007-67987-C02-02 and TEC2010-20224-C02-01. The associate editor coordinating the review of this manuscript and approving it for publication was Prof. Brian Culshaw.

The authors are with the Department of Electrical and Electronic Engineering, Public University of Navarra, Pamplona, Navarra 31006, Spain (e-mail: montserrat.fernandez@unavarra.es; dolier@unavarra.es; ander.zornoza@unavarra.es; rosa.perez@unavarra.es; silvia.diaz@unavarra.es; cesar.elosua@unavarra.escba@unavarra.es; alayn.loayssa@unavarra.es; mla@unavarra.es).

Color versions of one or more of the figures in this paper are available online at http://ieeexplore.ieee.org.

Digital Object Identifier 10.1109/JSEN.2011.2131128

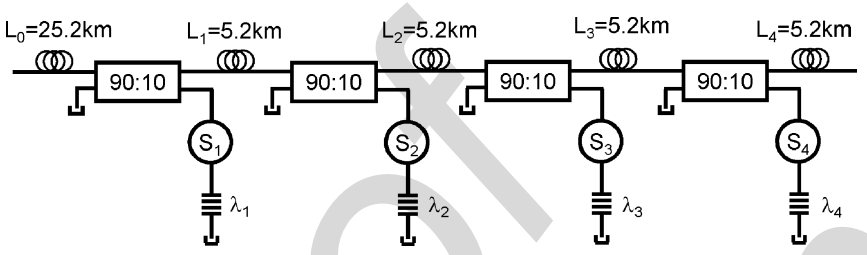

Fig. 1. Previous single bus topology.

a suitable technology for doing these tasks. These kinds of sensors are able to measure a number of environmental parameters [6] or gases [7].

Second, it is necessary to choose between "point," "quasi-distributed," and "distributed" sensors. The fiber optic point sensors are enabled to measure at specific points in the structure. A quasi-distributed sensor system is composed of different point sensors multiplexed in a fiber. Finally, distributed fiber optic sensors are particularly suitable for large structural monitoring applications since all the segments of an optical fiber act as sensors, and therefore the perturbations within various segments of the structure can be sensed [2], [3].

The three major distributed sensor techniques are: optical time domain reflectometry, sensors based on Raman, and Brillouin Scattering. The most appropriate technique for distributed sensing is based on Brillouin scattering because it provide simultaneous information of temperature and strain along the length of a standard low-cost telecommunication optical fiber [3].

Brillouin scattering can be stimulated by the use of a probe wave; thus, higher signal-to-noise ratios are attainable. Moreover, long distance measurements are possible because standard low-loss single-mode fibers are used and optical amplification can be deployed to further enhance of the reach of these systems [4].

Furthermore, long-range sensing networks for point sensors are now possible using Er-doped fiber or Raman optical amplification [8], [9].

We have previously reported a long-range hybrid network with point and distributed Brillouin sensors using Raman amplification which offered a great advantage: the fiber length deployed to interconnect and multiplex point sensors also serves as distributed sensor [10]. Nevertheless, the sensor network with a single bus topology, shown in Fig. 1, presents high noise levels.

In this paper, we propose an experimentally demonstrate an improved sensor network for multiplexing the point sensors based on a double-bus topology. The low noise configuration offers a great improvement in the signal-to-noise ratio; thus, 


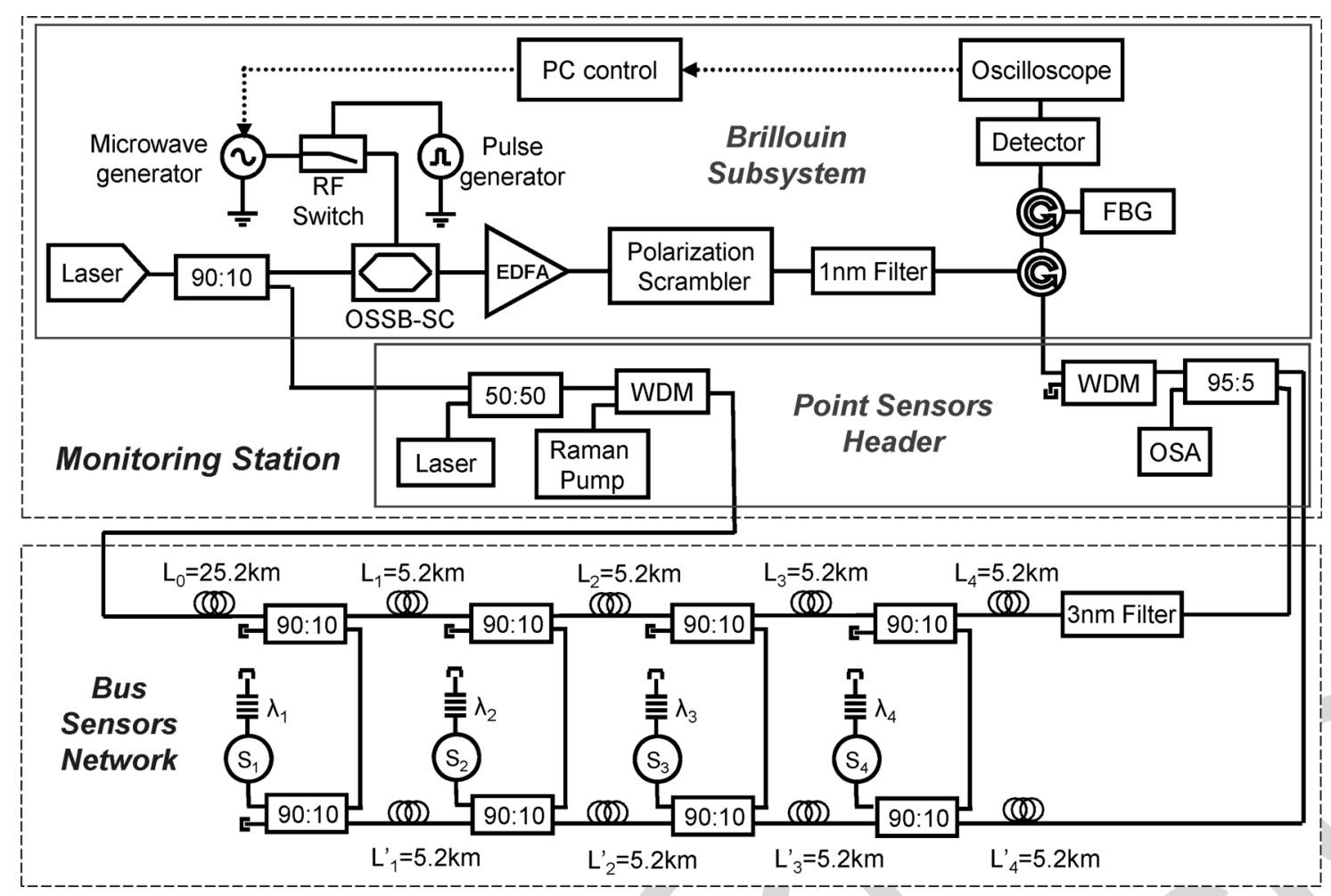

Fig. 2. Hybrid sensor network with point and distributed optical sensors.

the number of sensors to be multiplexed could increase or we could reach further distances.

\section{MethodOLOGY}

The experimental setup for the hybrid sensor network is shown in Fig. 2. It can be divided into two different parts: the sensor network itself and the monitoring station equipment.

The sensor network has a double-bus topology with a total length of $46 \mathrm{~km}$. Four-point sensors are wavelength-division multiplexed (WDM). Each sensor is addressed by a narrow-bandwidth FBG at a unique wavelength. Although the launched signals reaches all the sensors, the FBG ensures that each sensor returns only its information to the point sensor header after passing through the sensor a second time.

In the monitoring station are generated the signals needed for interrogation of the point sensors and for the Brillouin distributed measurements. The Brillouin measurement system is based on the Brillouin optical time-domain analysis (BOTDA) principle in which a pulsed pump and a continuous (CW) Sokes waves counterpropagate in the fiber under test (FUT).

A detailed explanation of the point sensors network subsystem and the Brillouin distributed sensing subsystem is presented in Sections II-A and II-B, respectively.

\section{A. Point Sensors Network Subsystem}

It is proposed a WDM network for point sensors multiplexing over $46 \mathrm{~km}$ of single-mode fiber used also for distributed sensing. The sensor network is composed by $46 \mathrm{~km}$ of standard single-mode fiber (SMF) and it has a double-bus topology based on [11] and [12]. It is used for both distributed sensing and point sensors multiplexing simultaneously.
The point sensors network subsystem includes eight directional couplers $(90: 10 \pm 0.3 \%$ ratios) to perform power distribution among the sensors. The distance between two adjacent couplers is about $5.2 \mathrm{~km} \mathrm{SMF}$. Both buses are identical with four couplers each one in order to wavelength-division multiplexing (WDM) the point vibration sensors.

The sensors, addressed by four fiber Bragg gratings centered at $\lambda_{1}=1539.8 \mathrm{~nm}, \lambda_{2}=1538.47 \mathrm{~nm}, \lambda_{3}=1533.9 \mathrm{~nm}$, and $\lambda_{4}=1535.36 \mathrm{~nm}$, are separated by $5.2-\mathrm{km}$ fiber spans. The grating wavelengths are located on the short wavelength side of the peak of Raman gain profile.

A Raman pump laser generates distributed amplification in the sensor bus using the first span of 25.2-km SMF, thus Raman gain is maximized in the bus and the fiber sensor network range is extended. The Raman pump power is launched into the fiber bus by using a fiber wavelength division multiplexer (WDM). It is able to launch up to $3.2 \mathrm{~W}$ into the single-mode fiber at $1445 \mathrm{~nm}$.

The signal used to interrogate the point sensors is a tunable laser (1460-1580 nm) with a spectral linewidth of $5 \mathrm{MHz}$.

The Raman pump and the signal are located at one end of the fiber outputs of the monitoring station. Another WDM is used at the other end of the bus to prevent the residual Raman pump from re-entering the monitoring station. Also the optical spectral analyzer (OSA) is located in the other head end. All the free terminations on the double-bus are refractive-index-matched to eliminate undesirable reflections.

The point vibration sensors multiplexed are based on fiberoptic taper sensors employed as transducers to measure mechanical vibrations. They show some advantages, as their simple fabrication process, low-cost and versatility in the number of mea- 
surable parameter [13]. The tapers were implemented by elongating the fiber during a fusion process, [14]. The transmitted optical power of the taper depends on its bending radius: as it decreases, the transmitted power decreases as well [15], so it can be used to vibrations just following the power variations. In this case, the optical signal crosses the taper back and forth, because the sensors are used in reflective configurations. Thus, there must be a compromise between the sensors' sensitivity, the induced losses and the maximum measuring length because although the sensors have double sensitivity the also have double losses.

\section{B. Brillouin Distributed Sensing Subsystem}

In the Brillouin distributed sensor, the output of a tunable laser source is first divided in two branches, so that the same light source is used for pump and stokes generation. In the upper branch a Dual Parallel Mach-Zehnder (DPMZ) biased at minimum transmission generates a pulsed optical single side band suppressed carrier modulation (POSSB-SC). The pulsing is achieved in the electrical domain using the RF shaping proposed in [16] so as to avoid leakage. The POSSB-SC signal is then applied to the sensing fiber as pump pulse after an active polarization scrambler is used to compensate the polarization sensitivity of SBS. Then, a 1-nm wide optical filter tuned to the wavelength of the POSSB-SC signal is inserted to reduce the amplified spontaneous emission (ASE) noise from the erbium-doped amplifier.

In the lower branch the laser output is directly used to provide the probe wave. No modulation at all is needed in this branch. In order to avoid any crosstalk from the point sensors interrogation system a fiber Bragg grating filter is used before detection. Note that the double-bus system makes no difference in the Brillouin sensing subsystem compared to the setup in [10].

\section{EXPERIMENTAL RESULTS}

It is experimentally demonstrated the hybrid double-bus network with point and distributed Brillouin sensors using Raman amplification following the design explained in previous section.

\section{A. Point Sensors Measurements}

In this subsection, we show the measurements obtained utilizing the point sensor inside the multiplexing network.

The fiber-optics sensors were fabricated as was explained in [10]. When vibration is applied to these taper sensors, the fiber is bent in the stretched area, giving a curvature's change, and then, a variation in the transmitted power. To characterize the sensor's response, we applied vibrations of different frequency, from $0.01 \mathrm{~Hz}$ to $100 \mathrm{~Hz}$; amplitude, from $0.025 \mathrm{~mm}$ to $3.25 \mathrm{~mm}$, and waveform, sinusoidal, square, triangular, and sawtooth waves. The results can be compared to the ones offered by commercial vibrometers with $\mathrm{mm} / \mathrm{s}$ RMS units [10].

Fig. 3(a) presents the spectrum for the four point sensors when Raman pump power is $1 \mathrm{~W}$. Amplification of the Brillouin signal and the signal used to interrogate the point sensors have been taking into account to select the optimum pump power. The output reflected signals reached $-24 \mathrm{dBm}$ and an OSNR of $55 \mathrm{~dB}$.
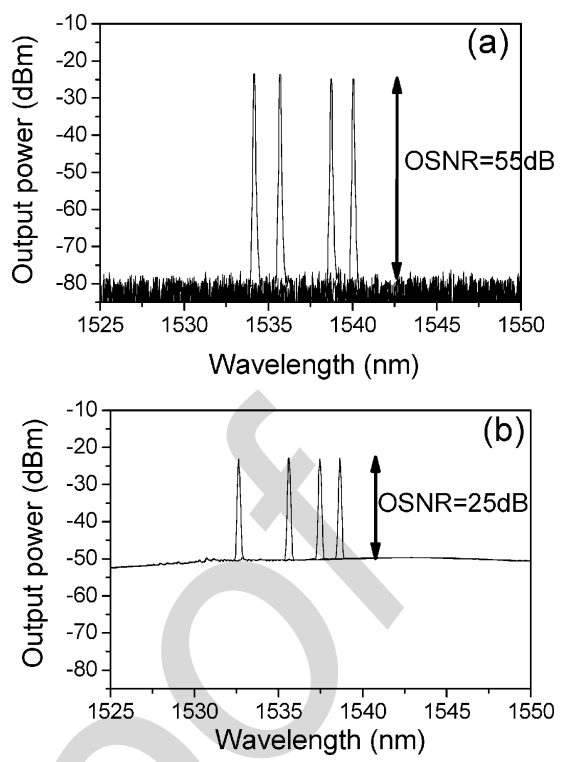

Fig. 3. Optical spectrum for the four point sensors. (a) Double-bus topology. (b) Single-bus topology.

The advantages of optical amplifier inclusion in sensor multiplexing networks are well-known. However, optical amplifier also involves constraints as a new noise source: amplified spontaneous scattering (ASS) if Raman amplification is used. For this reason, to optimizing sensor multiplexing networks behavior will be necessary to deplete the ASS. The double-bus topology presented obeys this important requirement.

Although the double-bus topology is more complicated than the used in a previous work where the sensor network was composed by a single bus [10], it offers a great appeal: it decrease the ASS noise level $30 \mathrm{~dB}$ below the values achieved by the single-bus topologies. The reduction of the ASS results in a great improvement of the OSNR. The single bus topology offers an OSNR of $27 \mathrm{~dB}$, while the double-bus topology OSNR reaches $55 \mathrm{~dB}$ as it is shown in Fig. 3. This enhancement is reached because in the single-bus topology, the backward amplified spontaneous-scattering (ASS) was co-propagating with the modulated signal and both were coupled to the OSA; however, with the double-bus topology the forward ASS propagates in the contrary direction than the signal, and thus only a bit amount of ASS reaches the OSA. Thus, with this low-noise configuration the number of sensors that could be multiplexed increases.

The main design parameter of the point sensors is the loss induced by the taper, which determines the sensitivity and the dynamic range. The losses induced by the sensor are $1.75 \mathrm{~dB}$, which were doubled in the experimental setup because the signal passes through each sensor twice, so the maximum losses are $3.5 \mathrm{~dB}$.

The double-bus topology has the advantage of obtaining an improvement in signal-to-noise ratio; thus, the dynamic range increases. The OSNR of this configuration reaches $55 \mathrm{~dB}$ : keeping in mind that the losses induced by the sensor are doubled because the signal passes twice through it, the insertion losses together with the dynamic range could be up to $27.5 \mathrm{~dB}$. For instance, in the case that the insertion losses were $3.5 \mathrm{~dB}$ to enhance the sensitivity, the dynamic range of the sensor could 


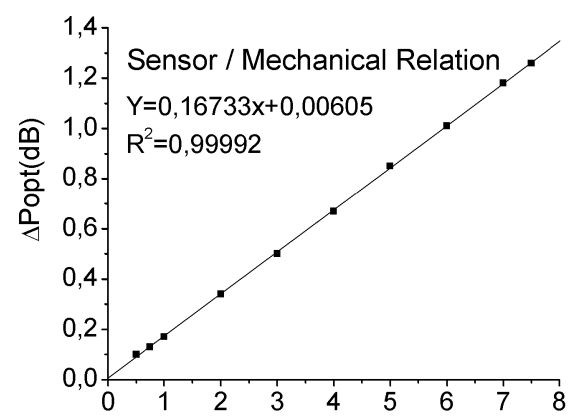

Peak to peak Vibration Amplitude, Dpp (mm)
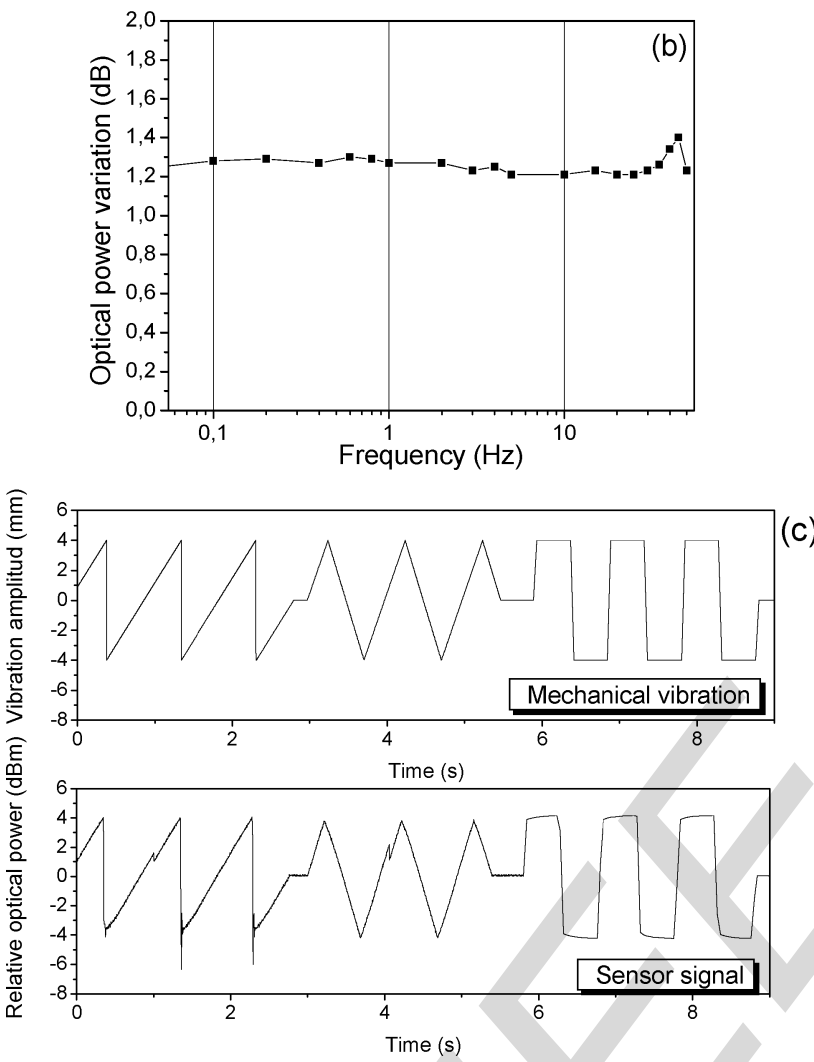

Fig. 4. Sensor's frequency, amplitude, and waveform responses for a sensor.

be up to $24 \mathrm{~dB}$ : in our case, they are $1.3 \mathrm{~dB}$ as a consequence of the design parameters of the sensor. For this reason, we could use sensor with higher dynamic range, add more point sensors in the network or reach further distances.

We have characterized the sensor behavior regarding two main parameters: frequency and amplitude response. To measure the frequency response, a sinusoidal wave with $6.5 \mathrm{~mm}$ peak-to-peak variation at different frequencies was applied. To analyze the amplitude response, a $45-\mathrm{Hz}$ vibration frequency was used and the amplitude was varied from 6.5 to $0.05 \mathrm{~mm}$ peak-to-peak. Fig. 4(a) shows that there is a linear relationship between the amplitude of the vibration and the variation in the sensor's signal. Regarding to the frequency response [Fig. 4(b)], it shows a resonance peak around $50 \mathrm{~Hz}$, so it might be used to operate with frequency below $30 \mathrm{~Hz}$. Fig. 4(c) depicts as different waveforms can be deployed as excitation signals: the response from the sensor follows the vibration signal from the vibrator.
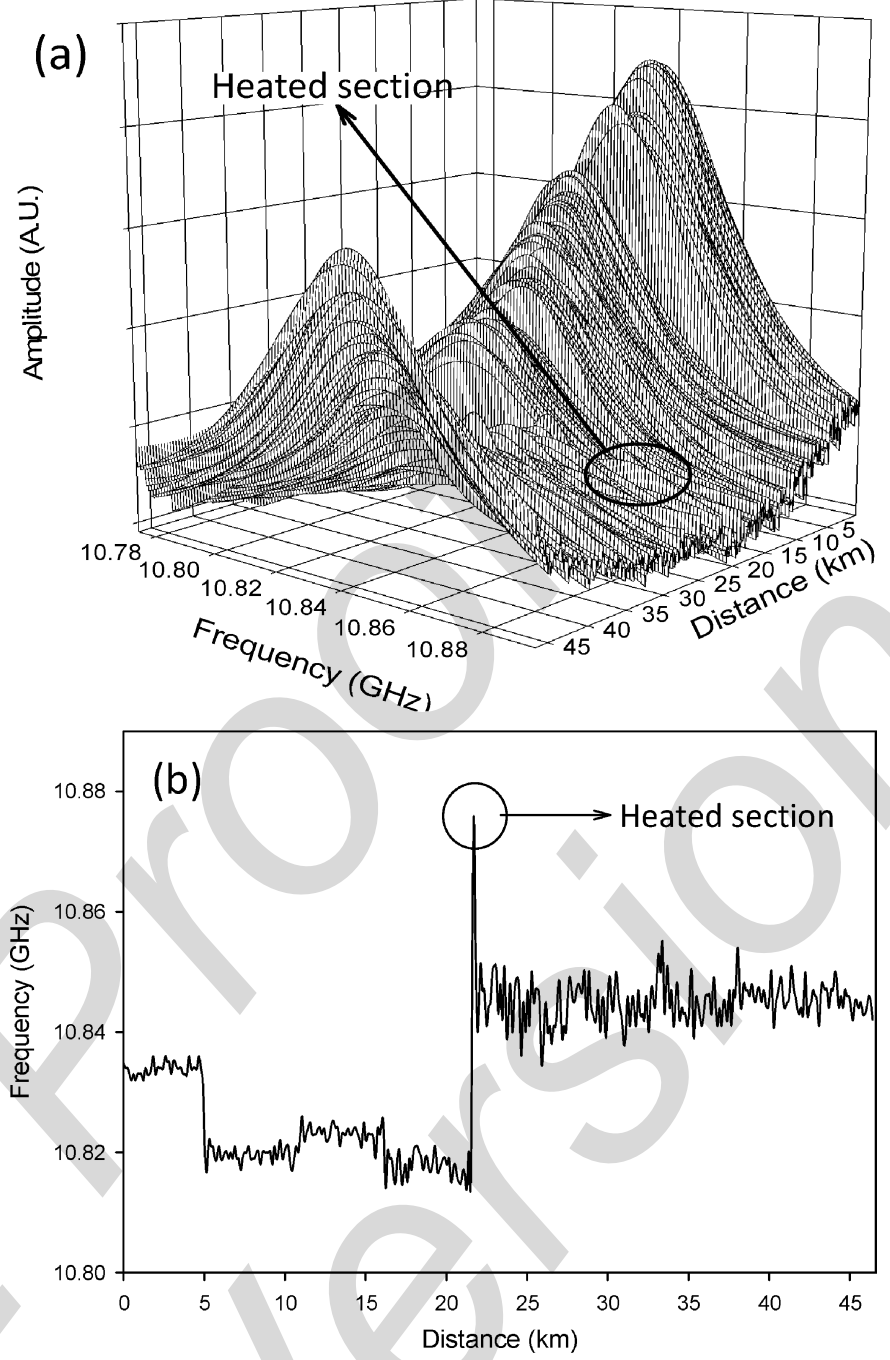

Fig. 5. (a) Distributed measurement of the Brillouin spectra. (b) Brillouin frequency shift of the whole fiber.

\section{B. Brillouin Distributed Temperature Measurements}

In the Brillouin distributed sensor we performed distributed temperature measurements having a $3-\mathrm{m}$ resolution. $200 \mathrm{~m}$ of the fiber at a distance of $25 \mathrm{~km}$ of the beginning were placed in a climatic chamber at $55^{\circ} \mathrm{C}$ while the rest was held at room temperature. The RF was swept at 1-MHz steps and the traces were averaged 4000 times. The distributed measurement of the Brillouin spectra is shown in Fig. 5(a) and the Brillouin frequency shift of the whole fiber are shown in Fig. 5(b). Different types of fibers used in the bus sensors network and heated section are clearly distinguishable with a resolution of $4{ }^{\circ} \mathrm{C}$. This resolution was calculated in the worst case scenario: in the middle of the fiber, where the pump pulse power is minimum due to propagation loss and Raman amplification is not still noticeable.

\section{CONCLUSION}

A 46-km double-bus hybrid network that integrates point and distributed Brillouin sensors and uses Raman amplification has been implemented. The use of Raman amplification in BOTDA distributed sensors serves to compensate branching and fiber losses in the network and provides a spatial resolution of $3 \mathrm{~m}$. 
Furthermore, the simultaneous Raman amplification of the signals from the low-frequency (from 0.1 to $50 \mathrm{~Hz}$ ) vibration point sensors permits to place them as far as the end of the fiber bus at $46 \mathrm{~km}$ away from the interrogation equipment overcoming low power levels or backscattering noise problems.

\section{ACKNOWLEDGMENT}

The author would like to thank D. Leandro for his help.

\section{REFERENCES}

[1] H. -. Li, D. -. Li, and G. -. Song, "Recent applications of fiber optic sensors to health monitoring in civil engineering," Eng. Struct., vol. 26, pp. 1647-1657, 2004.

[2] E. Tapanes, "Fibre optic sensing solutions for real-time pipeline integrity monitoring," in Proc. Austral. Pipeline Ind. Assoc. Nat. Conv., 2001.

[3] C. Bariáin, I. R. Matías, F. J. Arregui, and M. López-Amo, "Experimental results towards development of humidity sensors by using a hygroscopic material on biconically tapered optical fibre," in Proc. SPIE - Int. Soc. Opt. Eng., Beijing, China, 1998, vol. 3555, pp. 95-105.

[4] C. Elosua, I. R. Matias, C. Bariain, and F. J. Arregui, "Volatile organic compound optical fiber sensors: A review," Sensors, vol. 6, pp. 1440-1465, 2006.

[5] M. Niklès, "Fibre optic distributed scattering sensing system: Perspectives and challenges for high performance applications," in Proc. SPIE - Int. Soc. Opt. Eng., Naples, Italy, 2007, vol. 6619, 66190D.

[6] J. M. Lopez-Higuera, Handbook of Optical Fibre Sensing Technology. New York: Wiley, 2002.

[7] M. N. Alahbabi, Y. T. Cho, and T. P. Newson, "150-km-range distributed temperature sensor based on coherent detection of spontaneous Brillouin backscatter and in-line Raman amplification," J. Opt. Soc. Amer. B, vol. 22, pp. 1321-1324, 2005.

[8] S. Diaz, S. Abad, and M. Lopez-Amo, "Fiber-optic sensor active networking with distributed erbium-doped fiber and Raman amplification," Laser Photon. Rev., vol. 2, pp. 480-497, 2008.

[9] S. Diaz, G. Lasheras, and M. Lopez-Amo, "WDM bi-directional transmission over $35 \mathrm{~km}$ amplified fiber-optic bus network using Raman amplification for optical sensors," Opt. Exp., vol. 13, pp. 9666-9671, 2005.

[10] A. Zornoza, R. A. Pérez-Herrera, C. Elosúa, S. Diaz, C. Bariain, A. Loayssa, and M. Lopez-Amo, "Long-range hybrid network with point and distributed Brillouin sensors using Raman amplification," Opt. Exp., vol. 18, pp. 9531-9541, 2010.

[11] S. Abad, M. López-Amo, J. M. López-Higuera, D. Benito, A. Unanua, and E. Achaerandio, "Single and double distributed optical amplifier fiber bus networks with wavelength-division multiplexing for photonic sensors," Opt. Lett., vol. 24, pp. 805-807, 1999.

[12] V. Montoya, M. López-Amo, and S. Abad, "Improved double-fiber-bus with distributed optical amplification for wavelength-division multiplexing of photonic sensors," IEEE Photon. Technol. Lett., vol. 12, no. 9, pp. 1270-1272, Sep. 2000.
[13] I. R. Matías, C. Fernández-Valdivielso, F. J. Arregui, C. Bariáin, and M. López-Amo, "Transmitted optical power through a tapered singlemode fiber under dynamic bending effects," Fiber Integr. Opt., vol. 22, pp. 173-187, 2003.

[14] P. Datta, I. Matías, C. Aramburu, A. Bakas, M. López-Amo, and J. M. Otón, “Tapered optical-fiber temperature sensor," Microw. Opt Technol Lett., vol. 11, pp. 93-95, 1996.

[15] F. J. Arregui, I. R. Matias, C. Bariain, and M. Lopez-Amo, "Experimental design rules for implementing biconically tapered single mode optical fibre displacement sensors," in Proc. SPIE, 1998, vol. 3483, pp. $164-168$.

[16] A. Zornoza, D. Olier, M. Sagues, and A. Loayssa, "Brillouin distributed sensor using RF shaping of pump pulses," Meas. Sci. Technol., vol. 21, no. 9 , p. 094021,2010

Montserrat Fernandez-Vallejo, photograph and biography not available at the time of publication.

David Olier, photograph and biography not available at the time of publication.

Ander Zornoza, photograph and biography not available at the time of publication.

Rosa Ana Perez-Herrera, photograph and biography not available at the time of publication.

Silvia Diaz, photograph and biography not available at the time of publication.

Cesar Elosua, photograph and biography not available at the time of publication.

Candido Bariain, photograph and biography not available at the time of publication.

Alayn Loayssa, photograph and biography not available at the time of publication.

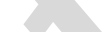
Manuel Lopez-Amo, photograph and biography not available at the time of
publication. 